\title{
Milliseconds matter: temporal order of visuo-tactile stimulation affects the ownership of a virtual hand
}

Conference or Workshop Item

Accepted Version

Zoulias, I., Harwin, W., Hayashi, Y. and Nasuto, S. (2016) Milliseconds matter: temporal order of visuo-tactile stimulation affects the ownership of a virtual hand. In: Eurohaptics 2016, 4-7 July, London, pp. 479-489. Available at http://centaur.reading.ac.uk/66337/

It is advisable to refer to the publisher's version if you intend to cite from the work. See Guidance on citing.

Published version at: http://dx.doi.org/10.1007/978-3-319-42324-1_47

Publisher: Springer International Publishing

All outputs in CentAUR are protected by Intellectual Property Rights law, including copyright law. Copyright and IPR is retained by the creators or other copyright holders. Terms and conditions for use of this material are defined in the End User Agreement. 


\section{www.reading.ac.uk/centaur}

\section{CentAUR}

Central Archive at the University of Reading

Reading's research outputs online 


\title{
Milliseconds matter: Temporal order of visuo-tactile stimulation affects the ownership of a virtual hand
}

\author{
Ioannis Dimitrios Zoulias ${ }^{1}$, William Seymour \\ Harwin $^{1}$, Yoshikatsu Hayashi ${ }^{1}$, and Slawomir Jaroslaw Nasuto ${ }^{1}$ \\ School of Sysems Engineering, University of Reading, UK \\ i.d.zoulias@pgr.reading .ac .uk
}

\begin{abstract}
The sense of body ownership, that one's body belongs to oneself, is a result of the integration of different sensory streams. This sense however is not error-free; in 1998 Botvinick and Cohen [3] showed the rubber hand illusion (RHI), an illusion that made a subject feel a rubber hand as their own. An important factor to induce the illusion is the timing of the applied visual and tactile stimulation to the rubber hand. Temporal delays greater than 500ms eliminate the illusory ownership. This study investigates previously unexplored small delays between stimulation modalities and their effect for the perception of the RHI. Through a virtual reality setup of the RHI paradigm, it is shown that small delays can significantly alter the strength of the illusion. The order of the sensory modality presented plays a catalytic role to whether or not the inter-modal delay will have an effect on the illusion's strength.

Keywords: Virtual Hand Illusion · Multisensory integration · Temporal Order Judgement · Virtual Reality
\end{abstract}

\section{Introduction}

Our perceptual capacity for understanding the external world, as well as the awareness of our self depend on a coherent stream of information flowing from the bodily senses [15]. The integration of the senses plays a pivotal role in our conscious experience [11]. Our ability to identify that our body belongs to us, that is our sense of body ownership, is one such experience that is regulated by the integration of sensory modalities [2,5,6,21].

However, human senses do not always present us with a truthful representation of the environment; the senses can be tricked both at the low level (e.g the Muller-Lyer optical illusion [13]) and at a higher cognitive level. The rubber hand illusion (RHI) falls into the latter category. It is an illusion of ownership that makes a person believe that an inanimate object constitutes a part of their body [3]. To achieve the RHI, a subject is shown a rubber replica of a human hand while their real hand is occluded behind a screen. The subject's hand is then stimulated using a brush while at the same time the rubber hand visible 
to them is "stimulated" in the same fashion. After a short time the subject's perception of body ownership is shifted such that they perceive the rubber hand as part of their body. This effect has been shown to produce a drift of the perceived position of the real hand (proprioceptive drift) and a feeling of threat over the rubber hand $[3,8,14]$. The RHI has been extensively studied since it was first discovered and with the technological advances of virtual reality in the last decade, the illusion has been extended to include a virtual world paradigm; the virtual hand illusion $[18,19]$.

One important factor to elicit the RHI is the application of congruent stimulation; applying asynchronous stimulation of the visual and tactile cues can be detrimental to the illusion [20]. Studies have found that the illusion disappears when there are large time differences $(>500 \mathrm{~ms})$ between the tactile stimulation of the hand and the visual cue on the fake hand. However, few studies have looked at smaller delays $[1,16,17]$.

A discrepancy is observed when the above time periods are contrasted with the reports on temporal order judgement and sensory simultaneity, indicating that delays smaller than $100 \mathrm{~ms}$ across multiple modalities can be detected by the human senses [11], with visuo-tactile noticeable differences ranging between $30 \mathrm{~ms}$ to $65 \mathrm{~ms}[7,9,10,12]$. Insights from those results can lead to a better understanding of the timings involved in sensory integration that underpin cognitive processes related to ownership. They also have applications in the design of virtual environments; to discern the optimal range of delays in VR settings with multi-sensory stimulation for achieving an elevated experience of presence.

This paper discusses results from a novel, ecologically valid, virtual hand illusion experiment. The experiment explored the range of stimulation delays from visuo-tactile sensory modalities that a human participant cannot detect as asynchronous. The aim was to quantify the effect of small temporal differences between visual and tactile stimulation on the perception and strength of the virtual hand illusion.

\section{Methods}

\subsection{Participants}

Twenty seven healthy participants ( 7 females) aged $18 \mathrm{yr}$ to $28 \mathrm{yr}$ (Mean age: $20 \mathrm{yr}$ ) were recruited from the University of Reading. The experimental design was reviewed and approved by the University of Reading Ethics Committee. All subjects gave written consent to participate in the study and were compensated $£ 10$ for their time. Participants were right handed with normal or corrected to normal vision. One participant was excluded from the analysis as an outlier due to reporting no ownership in any experimental condition.

\subsection{Experimental procedure}

At the beginning of each session, the participants were briefed about the experiment and were given a demonstration of the virtual environment, tactile actuator 


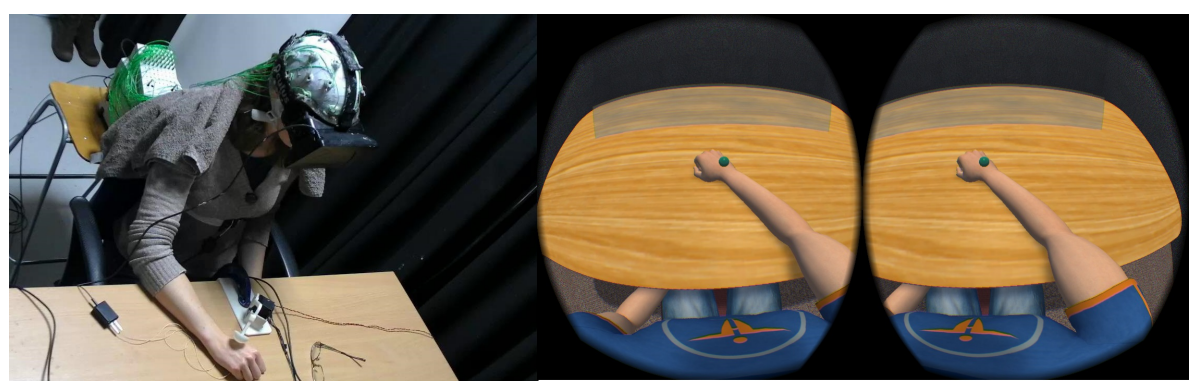

Figure 1. Experiment Setup. Left: Subject wearing the HMD, recording equipment and controller (under the desk). Mounted on the desk is the robotic actuator with the 3D printed ball attachment for delivering the tactile cue on the participant's hand. Right: 2D representation of the visual stimulation from left and right eye view.

and input system for the questionnaire in the VR. The virtual environment was designed using the Unity $3 \mathrm{D} \cap$ game engine and was modelled on the physical room in which the experiment was conducted, with a desk, chair, and the subject's avatar in a sitting position. The avatar used to represent the subject's own body was created using the MakeHuman $\AA$ parametric modelling software and was gender matched to the participant. Visual stimulation was presented using the Oculus Rift@ DK1 head mounted display (HMD); the device's suitability for experiment was tested in a previous study [23]. Answers in the way of a 7 point Likert scale ("strongly disagree" to "strongly agree") to questions presented in the VR were recorded using a microsoft Xbox@ controller and are presented in this paper in a scale from -3 to +3 corresponding to "strongly disagree" and "strongly agree" respectively with 0 representing "neutral". The actuator used to present the tactile stimuli was an MX-64A Dynamixel@ digital servo with a custom made 3D printed ball attachment. After briefing, the participants were prepared for EEG, EMG and GSR recordings; data not shown. Timing accuracy for the visuo-tactile stimulus delivery system was calibrated to be within $16.6 \mathrm{~ms}$; a single frame of the visual stimulation from the $60 \mathrm{~Hz}$ refresh rate of the HMD.

The participants would take part in two experiments, this paper outlines the result of one. Each experiment lasted $\sim 30 \mathrm{~min}$ with a 5 -10min break between each experiment. The order of the experiments was randomly chosen a priori to avoid order bias, and both experiments started first an equal number of times. The participant wore the HMD throughout the experiment. During the break between experiments the HMD was switched off to avoid fatigue. The experimental setup can be seen in Figure 1. The answers from the VR questionnaires were imported in MATLAB@ and were analysed first using a Kruskal-Wallis parametric test and post-hoc using a non-parametric two-tailed Wilcoxon rank sum test. All reported P-values have been corrected for multiple comparisons using a Bonferroni-Holm test. 


\subsection{Stimuli}

Each trial consisted of a 10s preparation period pre-stimulus and a stimulation period lasting $\sim 45$ s during which the participant was tapped 20 times on the right hand. A tap had both a tactile cue (a plastic ball attached to the actuator) and and a visual cue (a virtual ball touching the hand). Each tap lasted for $400 \mathrm{~ms}$ with a random period of $800 \mathrm{~ms}$ to $1100 \mathrm{~ms}$ between taps. At the end of each trial the participant was asked to report their feeling of ownership and their perception of simultaneity and temporal order of the visual and tactile stimulation (see Table 1). There were 7 conditions in the experiment derived by the delay onset between the visual and tactile stimulation of the hand. The first 5 conditions tested small delay values in the range of $-128 \mathrm{~ms}$ to $+128 \mathrm{~ms}$ with $64 \mathrm{~ms}$ increments $(-128 \mathrm{~ms},-64 \mathrm{~ms}, 0 \mathrm{~ms},+64 \mathrm{~ms},+128 \mathrm{~ms})$. Those increments were designed to allow for no overlap between conditions based on the limitation imposed by the $16.6 \mathrm{~ms}$ timing accuracy of co-stimulation. Negative values correspond to leading with visual stimulation and $0 \mathrm{~ms}$ denotes the synchronous condition. The 6th condition was a negative control condition with a delay of $+500 \mathrm{~ms}$. Finally, in the 7 th condition there was no fixed delay during the trial; for each tap, the delay between visual and tactile stimulation was randomly sampled from the pool of delays from the other conditions and could be positive, negative or synchronous. This condition aimed to to decrease the effect from sensory recalibration [4] by introducing a random stimulation pattern. All 7 conditions were randomly arranged and repeated once within a block of 7 trials with the experiment lasting a total of 3 blocks.

Table 1. Questionnaire asked at the end of each block (Q.1 - 7)

Virtual hand delays experiment questions

1. I felt as if the hand I saw in the virtual world might be my hand

2. The hand I saw was the hand of another person

3. The hand I saw resembles my own hand in terms of shape, skin tone, freckles, etc.

4. I felt the ball touch my hand at the SAME TIME as I saw the ball touch my hand

5. I felt the ball touch my hand BEFORE I saw the virtual ball touch my hand

6. I felt the ball touch my hand AFTER I saw the virtual ball touch my hand

7. I find it difficult to report the order of seeing and feeling the ball touch my hand

\section{Results}

\subsection{Perception of delays}

To analyse the perception of temporal order, each pair of conditions with equal but opposite delays (e.g. $-128 \mathrm{~ms}$ vs $+128 \mathrm{~ms}$ ) was tested for the difference between the answers for each of the three timing questions (Q.4-6, Table 1). Additionally, 
all of the delay conditions $(-128 \mathrm{~ms},-64 \mathrm{~ms},+64 \mathrm{~ms},+128 \mathrm{~ms})$ were tested against the synchronous condition (0ms).

For the perception of simultaneity, the results showed that when tactile stimulation was leading, participant reports were significantly different from the synchronous condition (Q.4 P $<0.05$ for $+64 \mathrm{~ms}, \mathrm{P}<0.001$ for $+128 \mathrm{~ms}$ ), see Figure 2. A change in their perception of the stimulation simultaneity is illustrated by a shift from agreeing that visual and tactile stimuli were presented synchronously to answers varying between "somewhat agree" and "neutral". In the negative control, participant reported no simultaneity (median = "strongly disagree").

The comparison of results from Q.4-5 also supports the shift in perception of asynchrony in the tactile leading conditions. The $+64 \mathrm{~ms}$ condition yielded different responses from the synchronous condition $(\mathrm{Q} .4 \mathrm{P}<0.05)$ when participants were asked if tactile feedback preceded visual feedback (Figure 2). Conversely, in Q.5 no such shift is seen for the visual leading condition. The random condition was tested against the null hypothesis (and rejected; Q.4 P $=0.0039, \mathrm{z}=2.8877$ ) that it was drawn from a 0 median distribution (median = "neutral") with a median corresponding to "somewhat agree" for simultaneity.

Finally, the participants reported little difficulty in reporting most delays, with median equal to "somewhat disagree" for most conditions. The $+128 \mathrm{~ms}$ was significantly different both from the synchronous condition and its $-128 \mathrm{~ms}$ paired condition $(\mathrm{Q} .7 \mathrm{P}<0.01$ for $+128 \mathrm{~ms}$ vs $-128 \mathrm{~ms}$ and $\mathrm{P}<0.001$ for $+128 \mathrm{~ms}$ vs synchronous). In the same question the random condition reported significantly higher difficulty in identifying the delays compared to all other conditions.

\subsection{Ownership}

The same comparisons as above were made for the ownership questions (Q.1-2). Results from questions Q.1-2 showed that the experiment elicited the illusion in the participants (median = "agree" to "somewhat agree") during the synchronous condition and was significantly different from the negative control (Q.1 P $<0.001$, Figure 3 ). The difference between the $+128 \mathrm{~ms}$ and the synchronous conditions was shown to be significant (Q.1 P $<0.05$, Figure 3). Reports of disownership was shown to be significantly different only between the synchronous and control conditions (Q.2 $\mathrm{P}<0.001)$.

To further investigate the effect of the perception of delays a post hoc comparison was made within the $+128 \mathrm{~ms}$ condition. Trials were split between those with low perception of delay (when the answer to Q.4 was below "neutral") and those with a high perception of delay (where participants answered Q.4 with a statement of agreement). Trials from the two groups were then compared on their reported level of ownership. It was found that trials in which the participant could identify the temporal delay had a significantly different response of ownership level compared to trials during which participants had low perception of the delay $(\mathrm{P}<0.01$, Figure 4$)$. 

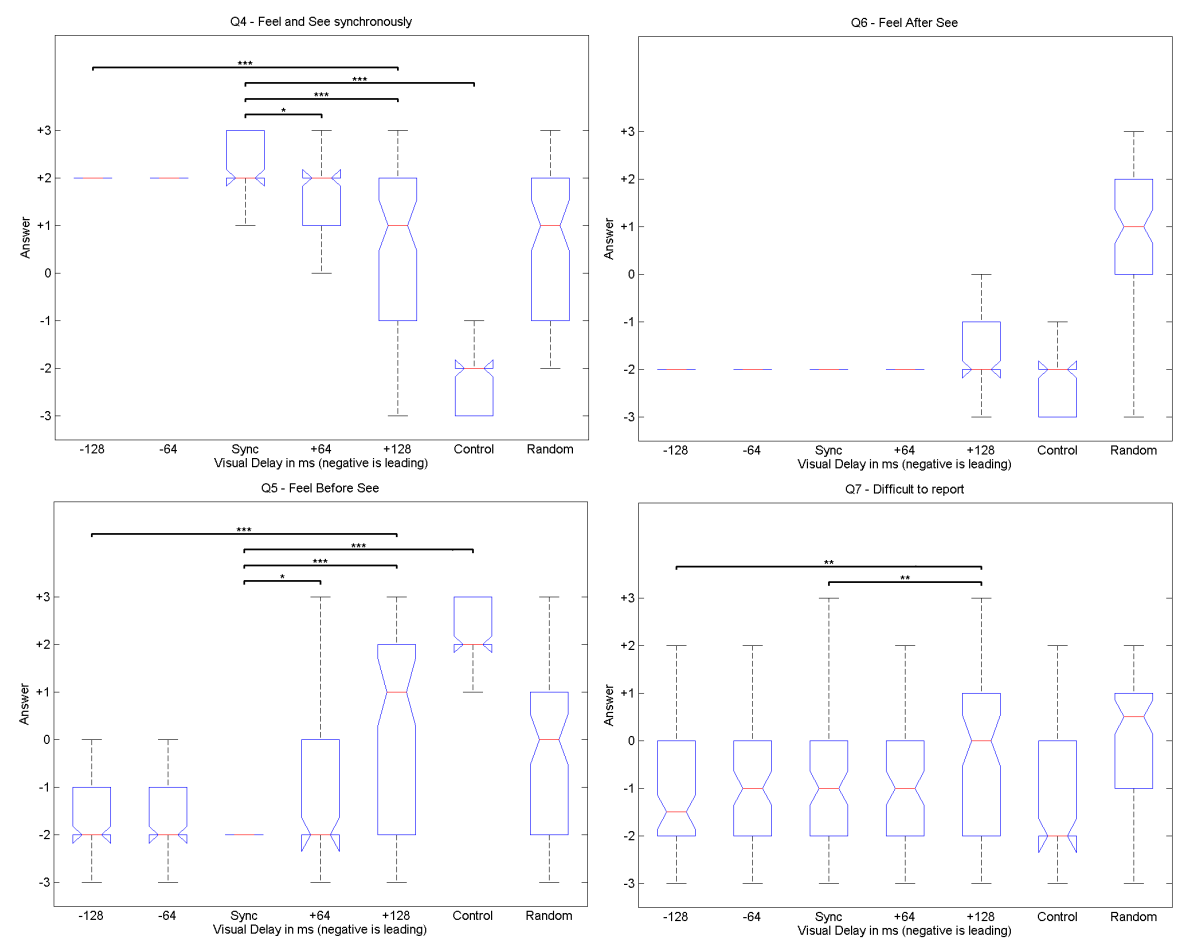

Figure 2. Temporal order judgement and synchrony. Top left: Responses to synchronous stimulation. Results for conditions with tactile stimulus lead show that participants were significantly more likely to notice a difference. Top right: Responses to feeling the tactile cue after the visual cue. Bottom left: Responses to feeling the tactile cue before the visual. Bottom right: Difficulty to respond to Q.4-Q.6. Answer values -3 to +3 correspond to 7 point Likert answers "strongly disagree" to "strongly agree". Negative conditions denote visual cue leading. Sync: Synchronous condition (0ms delays). Control: negative control $(+500 \mathrm{~ms}$ delay. Random: Random condition, each tap during the trial assigned randomly from all other available delays. $*, * *, * * *$ : corresponding to pvalues at $0.05,0.01$ and 0.001 significant level respectively. Red line is the median, top and bottom of boxes are the 75 th and 25 th percentile respectively, whiskers correspond to approximately $99 \%$. 

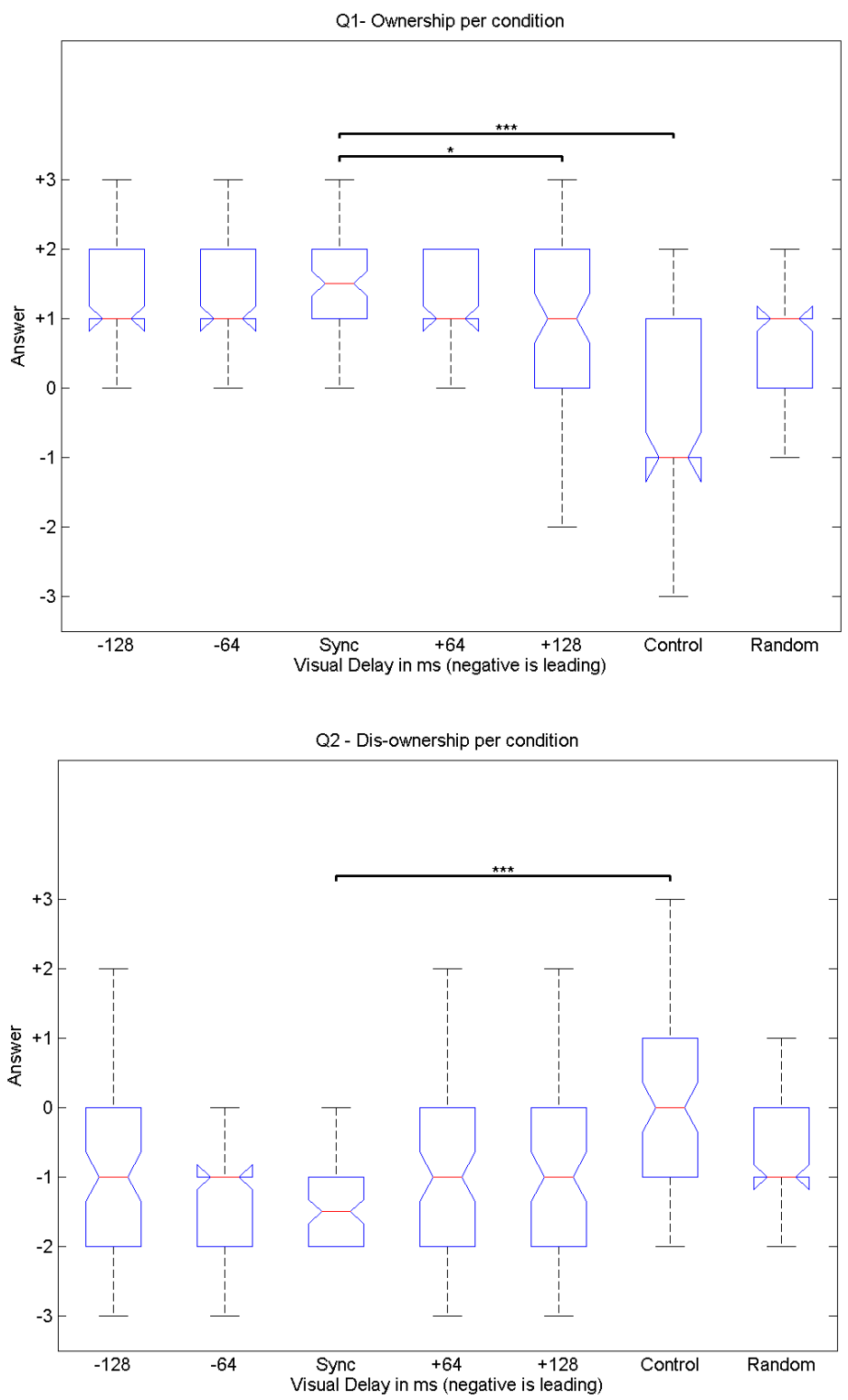

Figure 3. Ownership and dis-ownership. Top: Reports of ownership. Tactile cue leading by $128 \mathrm{~ms}$ produces significantly weaker illusion than the synchronous condition. Bottom: Reports of dis-ownership of the hand. The experiment was successful in evoking RHI. Red line is the median, top and bottom of boxes are the 75 th and 25th percentile respectively, whiskers correspond to approximately $99 \%$. 


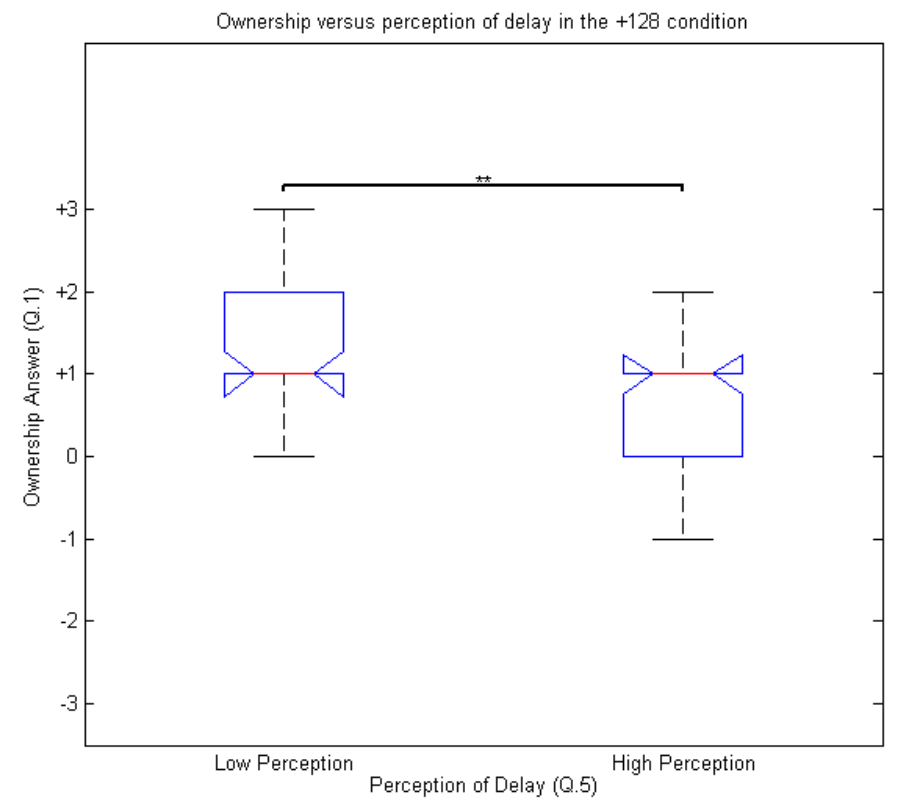

Figure 4. Effect on ownership from trials with high and low perception of delays. Using data from the $+128 \mathrm{~ms}$ condition trials were split by the subjects response of Q5; below "neutral" was grouped in low perception and above was grouped in high perception. The response on the level of the illusion is significantly lower for the group that perceived the delay. This supports the hypothesis that the perception of a delay between the visual and tactile stimulation has an effect on the reported level on ownership. Red line is the median, top and bottom of boxes are the 75 th and 25 th percentile respectively, whiskers correspond to approximately $99 \%$. 


\section{Discussion}

\subsection{Temporal order affects perception of synchrony}

Confirming results from the literature on audio-visual and audio-tactile temporal order $[7,9,11,12]$, this study suggests a visual lead for the perception of multisensory synchrony. As shown in Figure 2, no condition for which the visual stimulation is leading shows significant difference from the synchronous condition. Conversely, conditions with tactile lead are reported to be significantly different than the synchronous condition. The results from the random condition support this evidence; the null hypothesis would suggest that the responses should be balanced around the neutral response. Instead, the observed preference towards "somewhat agree" on simultaneity could be explained as a bias caused by a larger proportion of synchronously perceived taps due to the visual leading conditions.

It is important to note that the medians between conditions that show significantly different responses might not change; this is expected as the perception of the time delay will differ for each participant. Additionally, the repeated nature of the experiment, necessary in order to generate the RHI, could produce a sensory recalibration effect further pushing the perception towards synchrony $[4,10,22]$. However, the change in the range of responses and the trend seen in the data suggests that the window for failing to notice small asynchronies is much larger when vision is leading versus when tactile stimulation is leading. Furthermore, the higher variation in responses shows that as the delay between the visual and tactile stimulation increases, a larger population of subjects will perceive the asynchrony for tactile leading conditions.

\subsection{Effect of delays on ownership}

The results showed that the order of the stimulation not only has an effect on the window of asynchrony detection, but also on the perceived strength of the illusion. The small delays, although not sufficient to abolish illusory ownership, significantly decreased the perceived strength of the illusion. This suggests the possibility that the sense of ownership is not necessarily an all or none phenomenon, but rather it may be a graded experience. The possibility of different levels of ownership is further shown when looking at the responses of ownership within the $+128 \mathrm{~ms}$ condition. In this condition those participants who could spot the delay reported a lower level of ownership than those who didn't. Furthermore, the theory of a graded ownership has support from a previous study by Shimada et al[16] that looked at the effect of gradient delays to ownership.

An important note is on the size of the effects reported in this study. In the comparison between the synchronous and $+128 \mathrm{~ms}$ conditions on Q.1 there is a small effect size (Cliff's $d=0.2549$ ), while a medium effect is seen in the comparison between high and low delay perception (Cliff's $d=0.4214$ ). The small effect size could be explained by sensory recalibration; within a trial, a subject's perception of the time between the stimulation cues gets recalibrated 
to perceive the two cues as synchronous, hence the perceived difference between the conditions becomes smaller. Another possible explanation is that ownership is the result of the integration of many senses [2,6]; small inconsistencies within a subset of the responsible senses for ownership are compensated by agreement from the remaining set of the senses (e.g. a visual - proprioceptive agreement).

On the importance of the order of the stimulation on delay, a previous study by Bekrater-Bodmann[1] found no difference on ownership as a result of the order of sensory integration. In their experiment visual leading and tactile leading stimulation of $300 \mathrm{~ms}$ were compared with no significant difference found. A possible explanation for the discrepancy between that study and the results presented here is the magnitude of the stimulation delays. As shown in section 3.1 , visual leading delays of $128 \mathrm{~ms}$ are not perceived by the participants. However a larger visual leading delay of $300 \mathrm{~ms}$ would be perceived[9,12]. If the perception of delay is necessary to affect the level of ownership no significant effect would be seen between delays of $+/-300 \mathrm{~ms}$. Contrary, the order of stimulation would play a more significant role for delays of $+/-128 \mathrm{~ms}$ due to the difference induced by the order of stimulation in the perception of the delays. Further exploration of delays in the range of $100 \mathrm{~ms}$ to $300 \mathrm{~ms}$ could provide more evidence on the asymmetric boundary of perception of the delays and hence on the effect of stimulation order on the level of ownership.

Finally, a comment on the dis-ownership question (Q.2) is that a participant might not feel embodiment over the presented virtual hand and at the same time not feel that the virtual hand belongs to someone else either. Reporting RHI using questionnaires has limitations [21] and an objective measure from biophysical markers would be preferable for assessing the illusion.

\section{Conclusions}

This study has shown that small delays have a negative effect on the strength of the RHI when tactile stimulation precedes the visual cue. The results of this study suggest that when inter-modal stimulation delays are unavoidable, stimulation where visual cues are leading is preferable due to the stimulation time differences not being perceived. The former is applicable to any VR setting where ownership of the avatar is required.

Further work should be conducted to investigate stimulation lags in the range of $100 \mathrm{~ms}$ to $300 \mathrm{~ms}$ as identified by the results of this study. Furthermore, a future goal of this study is to suggest an objective method for identifying ownership usign biophysical data collected instead of questionnaires.

Acknowledgements. This research was supported by an EPSRC DTG grant

\section{References}

1. Bekrater-Bodmann, R., Foell, J., Diers, M., Kamping, S., Rance, M., Kirsch, P., Trojan, J., Fuchs, X., Bach, F., Çakmak, H.K., Maaß, H., Flor, H.: The Importance 
of Synchrony and Temporal Order of Visual and Tactile Input for Illusory Limb Ownership Experiences: An fMRI Study Applying Virtual Reality. PLoS ONE 9(1), e87013 (2014), http://dx.plos.org/10.1371/journal.pone.0087013

2. Blanke, O.: Multisensory brain mechanisms of bodily selfconsciousness. Nature reviews. Neuroscience 13(July), 556-71 (jul 2012), http://www.nature.com/doifinder/10.1038/nrn3292

3. Botvinick, M., Cohen, J.: Rubber hands 'feel' touch that eyes see. Nature 391(6669), 756 (feb 1998), http://www.ncbi.nlm.nih.gov/pubmed /9486643

4. Di Luca, M., Machulla, T.K., Ernst, M.O.: Recalibration of multisensory simultaneity: cross-modal transfer coincides with a change in perceptual latency. Journal of vision 9(12), 7.1-16 (2009)

5. Ehrsson, H.H., Holmes, N.P., Passingham, R.E.: Touching a rubber hand: feeling of body ownership is associated with activity in multisensory brain areas. The Journal of neuroscience : the official journal of the Society for Neuroscience 25(45), 10564-73 (nov 2005), http://www.pubmedcentral.nih.gov/articlerender.fcgi?artid=1395356

6. Ehrsson, H.: The Concept of Body Ownership and Its Relation to Multisensory Integration. In: Calvert, G.A., Spence, C., Stein, B.E. (eds.) The New Handbook of Multisensory Processes, chap. 43, pp. 775-792. MIT Press, Cambridge, Massachusetts (2012), http://130.237.111.254/ehrsson/pdfs/Ehrsson New Multisensory Handbook uncorrected proofs.pdf

7. Fink, M., Ulbrich, P., Churan, J., Wittmann, M.: Stimulus-dependent processing of temporal order. Behavioural processes 71(2-3), 344-52 (2006), http://www.sciencedirect.com/science/article/pii/S0376635705002627

8. González-Franco, M., Peck, T.C., Rodríguez-Fornells, A., Slater, M.: A threat to a virtual hand elicits motor cortex activation. Experimental Brain Research 232(3), 875-887 (mar 2014), http://www.ncbi.nlm.nih.gov/pubmed $/ 24337257$ http://link.springer.com/10.1007/s00221-013-3800-1

9. Kanabus, M., Szelag, E., Rojek, E., Poppel, E.: Temporal order judgement for auditory and visual stimuli. Acta Neurobiol Exp (Wars) 62(4), 263-270 (2002)

10. Keetels, M., Vroomen, J.: Temporal recalibration to tactile visual asynchronous stimuli. Neuroscience Letters 430(2), 130-134 (2008), http://linkinghub.elsevier.com/retrieve/pii/S0304394007011445

11. Keetels, M., Vroomen, J.: Perception of Synchrony between the Senses. In: Murray, M., Wallace, M.T. (eds.) The Neural Bases of Multisensory Processes, chap. Chapter 9. CRC Press/Taylor \& Francis (2012), http://www.ncbi.nlm.nih.gov/books/NBK92837/

12. Levitin, D.J., MacLean, K., Mathews, M., Chu, L., Jensen, E.: The perception of cross-modal simultaneity. International Journal of Computing Anticipatory Systems pp. 6-9 (2000)

13. Morgan, M., Hole, G., Glennerster, A.: Biases and sensitivities in geometrical illusions. Vision Research 30(11), 1793-1810 (jan 1990), http://linkinghub.elsevier.com/retrieve/pii/004269899090160M

14. Rohde, M., Di Luca, M., Ernst, M.O.: The Rubber Hand Illusion: Feeling of Ownership and Proprioceptive Drift Do Not Go Hand in Hand. PLoS ONE 6(6), e21659 (2011), http://dx.plos.org/10.1371/journal.pone.0021659

15. Romo, R., De Lafuente, V.: Conversion of sensory signals into perceptual decisions. Progress in Neurobiology 103, 41-75 (apr 2013), http://dx.doi.org/10.1016/j.pneurobio.2012.03.007 
16. Shimada, S., Fukuda, K., Hiraki, K.: Rubber Hand Illusion under Delayed Visual Feedback. PLoS ONE 4(7), e6185 (2009), http://dx.plos.org/10.1371/journal.pone.0006185

17. Shimada, S., Hiraki, K., Oda, I.: The parietal role in the sense of self-ownership with temporal discrepancy between visual and proprioceptive feedbacks. NeuroImage 24(4), 1225-32 (2005), http://www.ncbi.nlm.nih.gov/pubmed/15670700

18. Slater, M., Perez-Marcos, D., Ehrsson, H.H., Sanchez-Vives, M.V.: Towards a digital body: the virtual arm illusion. Frontiers in human neuroscience 2(August), 6 (jan 2008), http://www.pubmedcentral.nih.gov/articlerender.fcgi?artid=2572198

19. Slater, M., Perez-Marcos, D., Ehrsson, H.H., Sanchez-Vives, M.V.: Inducing illusory ownership of a virtual body. Frontiers in neuroscience 3(2), 214-20 (sep 2009), http://www.pubmedcentral.nih.gov/articlerender.fcgi? artid=2751618

20. Tsakiris, M., Haggard, P.: The Rubber Hand Illusion Revisited: Visuotactile Integration and Self-Attribution. Journal of Experimental Psychology: Human Perception and Performance 31(1), 80-91 (2005), http://doi.apa.org/getdoi.cfm?doi=10.1037/0096-1523.31.1.80

21. de Vignemont, F.: Embodiment, ownership and disownership. Consciousness and cognition 20(1), 82-93 (mar 2011), http://www.ncbi.nlm.nih.gov/pubmed/20943417

22. Vroomen, J., Keetels, M., de Gelder, B., Bertelson, P.: Recalibration of temporal order perception by exposure to audiovisual asynchrony. Cognitive Brain Research 22(1), 32-35 (2004), http://linkinghub.elsevier.com/retrieve/pii/S0926641004001946

23. Zoulias, I.D., Hayashi, Y., Harwin, W.S., Nasuto, S.J.: Visual Stimulation by the Oculus Rift (R) HMD to detect stronger motion imagery ERD. In: 7th International IEEE/EMBS Conference on Neural Engineering. Montpellier (2015), http://emb.citengine.com/event/ner-2015/paper-details?pdID $=3859$ 